\title{
MOLECULAR LINE OBSERVATIONS OF THE GALACTIC CENTER REGION
}

\author{
A. A. Stark, J. Bally, R. W. Wilson, and M. W. Pound \\ AT\&T Bell Laboratories \\ Crawford Hill Laboratory; Holmdel, NJ 07733
}

\begin{abstract}
A decade of galactic center observations at the Crawford Hill $7 \mathrm{~m}$ antenna is summarized. The galactic center region contains several hundred high-mass, highdensity molecular clouds with physical properties very different from clouds in the outer galactic disk. There is also a considerable amount of molecular gas not bound into clouds, but sheared by differential rotation into a molecular inter-cloud medium not seen elsewhere in the Galaxy. These observations can be explained by a combination of the tidal density limit and the virial theorem. The distribution of emission on the sky and in velocity suggests that most of the dense gas is confined to a 500 pc long ridge of emission which may be a dust lane along the central bar.
\end{abstract}

\section{Introduction}

We have observed about 200 gravitationally-bound molecular clouds in the inner kiloparsec of the Galaxy, each containing $10^{5}$ to $10^{6} \mathrm{M}_{\odot}$ of gas with an internal 1-dimensional FWHM velocity dispersion of $30 \mathrm{~km} \mathrm{~s}^{-1}$ and sufficient density to excite the $98 \mathrm{GHz}$ transition of CS (Bally et al. 1987, 1988). Most of the clouds are located in a thin layer in the galactic plane, but some are well above or below the plane (Bally et al. 1988). Many have velocities which deviate significantly from circular orbits around the galactic center, with radial motions that are comparable to the circular velocity (Bania, Stark and Heiligman 1986, Stark and Bania 1986, Heiligman 1987). In addition to these gravitationally-bound clouds, there is molecular gas which emits in the $\mathrm{J}=1 \rightarrow 0$ line of CO that is not bound into clouds - gas which forms a moderately dense but non-cloudy medium (Linke, Stark and Frerking 1981). By "non-cloudy", we mean that the gas is bound to the galaxy as a whole, but is not bound to any smaller region.

\section{Why Are These Clouds So Dense?}

We have identified gravitationally-bound clouds in the galactic center region in maps of the CS molecule (Bally et al. 1988). We do this because of a theoretical expectation that the CS emitting clouds in the galactic center region are approximately the same material as the gravitationally bound clouds. The CS J=2 $\rightarrow 1$ line is a density tracer: it is significantly excited into emission only when the ambient density $<n>22 \times 10^{4} \mathrm{~cm}^{-3}$. 


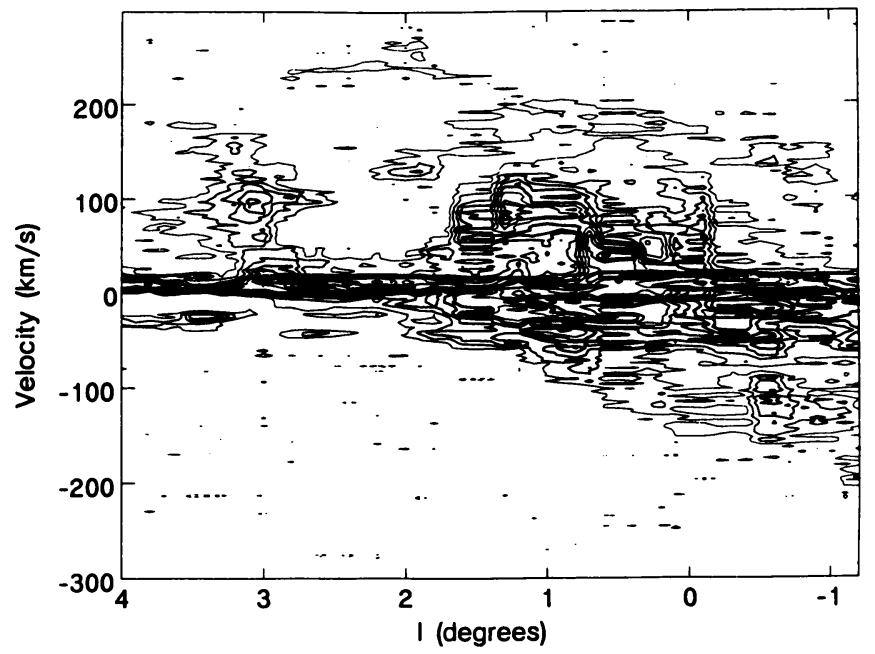

Fig. 1. Spatial-velocity diagram showing the velocity distribution of ${ }^{13} \mathrm{CO} \mathrm{J}=1 \rightarrow 0$ emission in the range $-0 . .^{\circ} \leq b \leq 0.5$ as a function of $l$. The data in the $6^{\prime}$ ${ }^{13} \mathrm{CO}$ survey have been averaged in $b$; the contours represent the average brightness of $1^{\circ}$ long strips in $b$ at constant $l$. Contour levels are equally spaced at $1 \mathrm{~K}$ intervals. Because of coarse sampling, this Figure shows some aliasing which is not present in Figure 2. Note that there is emission present at, e.g., $180 \mathrm{~km} \mathrm{~s}^{-1}$ between $l=0.0$ and $l=1 .{ }^{\circ} 7$, which does not appear in Figure 2: this gas has a density of about $10^{3} \mathrm{~cm}^{-3}$.

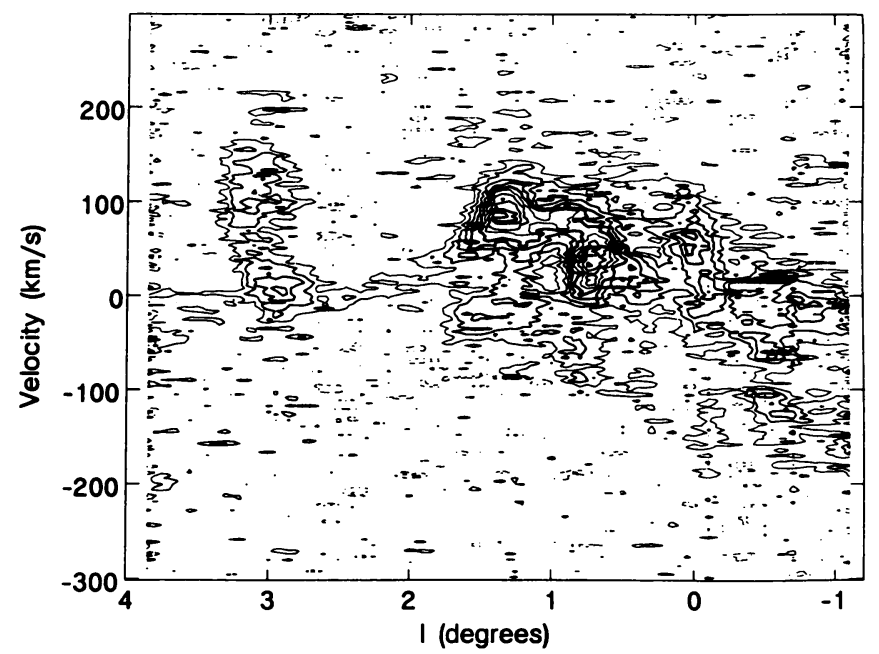

Fig. 2. Spatial-velocity diagram showing the velocity distribution of CS $\mathrm{J}=2 \rightarrow 1$ emission in the range $-0.4 \leq b \leq 0.4$ as a function of $l$. The data in the $3^{\prime}$ CS survey have been averaged in $b$; the contours represent the average brightness of $0 .{ }^{\circ} 8$ long strips in $b$ at constant $l$. Contour levels are equally spaced at $1 \mathrm{~K}$ intervals. 
In order for a cloud to be gravitationally bound, it must be sufficiently dense to withstand the tidal stress caused by the potential well of the Galaxy. In the galactic center region, this tidal density limit is approximately the same as the density threshold for exciting CS.

In a roughly spherical cloud of radius $r_{0}$, at a distance $R_{0}$ from the galactic center, consider the gravitational forces on a parcel of gas at a distance $r_{0}$ from the cloud center along a line towards the galactic center. If this parcel of gas is bound to the cloud, so that it follows a circular trajectory around the galactic center at a slower-than-orbital velocity, the cloud must exert a gravitational acceleration on it equal to $\operatorname{Tr}_{0}$, where

$$
\left.T \equiv-\frac{\mathrm{d}}{\mathrm{d} R}\left[\frac{v_{\text {circular }}^{2}}{R}\right)\right]_{R_{0}}+\frac{v_{\text {circular }}^{2}}{R_{0}^{2}} \approx 2 \frac{v_{\text {circular }}^{2}}{R_{0}^{2}}
$$

(the rotation velocity $v_{\text {circular }}$ is approximately independent of $R$ when $10 \mathrm{pc} \$ R \lessgtr 20 \mathrm{kpc}$ ). This acceleration is caused by the gravitational pull of the cloud, so that

$$
\frac{G M_{\text {cloud }}}{r_{0}^{2}} \gtrless T r_{0}
$$

and

$$
<\rho_{\text {cloud }}>\equiv \frac{M_{\text {cloud }}}{\left(4 / 3 \pi r_{0}^{3}\right)} \geq \frac{3 T}{4 \pi G} \approx \frac{3}{2 \pi G} \frac{v_{\text {circular }}{ }^{2}}{R_{0}^{2}} \approx 3.5 \times 10^{3} \text { a.m.u. } \mathrm{cm}^{-3}\left[\frac{500 \mathrm{pc}}{R_{0}}\right]^{2}
$$

The minimum average density of a cloud is a strong function of galactic radius, and clouds near the galactic center must be many times more dense than clouds in the solar neighborhood. Gravitationally bound clouds within about $200 \mathrm{pc}$ of the galactic center must be dense enough to excite the $\mathrm{CS}=2 \rightarrow 1$ line. Thus, maps in this line can be used to find clouds which are gravitationally-bound against the galactic tide. The galactic tide demands that the clouds be dense - they can be dense and small, or dense and large. One of the surprising things about the Galactic Center Region is that many of the clouds are dense and large, having masses as much as $10^{6} \mathrm{M}_{\odot}$.

\section{Why Do These Clouds Have High Internal Velocities?}

For stable gravitationally-bound clouds, the large observed line widths can be explained by the virial theorem which relates cloud density to cloud size and to the internal pressure. The dominant pressure term is turbulent pressure, characterized by a mean square velocity $\left\langle v^{2}\right\rangle$. Define a mean gravitational radius $\langle r\rangle$ such that the potential energy $W_{\text {cloud }} \equiv-G M_{\text {cloud }}^{2} /\langle r\rangle$. The relationship between $\langle r\rangle$ and $r_{0}$ is geometrical, depending on the distribution of mass within the cloud. For a spherical cloud where $\rho \propto r^{\alpha}$, with a sharp edge at $r_{0}$, the gravitational radius is related to the outer radius $\frac{r_{0}}{\langle r\rangle}=\frac{\alpha+3}{2 \alpha+5}$. The virial theorem can then be written as a requirement on the minimum internal velocity dispersion: 


$$
\left\langle v^{2}\right\rangle \approx \frac{G M_{\text {cloud }}}{\langle r\rangle}=\frac{4 \pi G}{3}\left\langle\rho_{\text {cloud }}\right\rangle\left(\frac{r_{0}}{\langle r\rangle}\right) r_{0}^{2} z 2 v_{\text {circular }}^{2}\left(\frac{r_{0}}{\langle r\rangle}\right)\left(\frac{r_{0}}{R_{0}}\right)^{2},
$$

where for $\rho_{\text {cloud }}$ we have substituted from the tidal density limit. This inequality does not impose a very stringent limit on the random velocities internal to outer-galaxy clouds. Most clouds in the outer galaxy have velocities 2 or 3 times bigger than this minimum. For the dense, large clouds in the galactic center, however, it says that a $30 \mathrm{pc}$ cloud 300 parsecs from the galactic center must have a FWHM linewidth of at least $37 \mathrm{~km} \mathrm{~s}^{-1}-\mathrm{a}$ typical linewidth for a galactic center cloud. Clouds as large as those we find in the galactic center must have large linewidths, or they will not remain in equilibrium and bound against the galactic tide.

\section{Molecular Dust Lanes in the Inner Galaxy}

A direct consequence of the above argument is that gas having a density less than that needed to bind the cloud against the galactic tide will be sheared by differential rotation into arcs and rings. Recent evidence for a nearly complete ring of gas surrounding the inner few parsecs of the Galaxy has been presented by Güsten et al. (1987). The "negative velocity arc" (Bally et al. 1988), between $l=-0.0^{\circ}$ and $l=0.0$ may be a sheared cloud. Comparison of $\mathrm{CS}$ and ${ }^{13} \mathrm{CO}$ longitude-velocity diagrams (Figures 1 and 2) show that there is low-level diffuse ${ }^{13} \mathrm{CO}$ emission between CS clouds. Linke, Stark and Frerking (1981) found that toward Sgr A and Sgr B2 continuum sources, this gas appears in emission in the $\mathrm{CO}$ lines, but appears in absorption in a number of high-excitation molecules including CS. To achieve this excitation condition, the density of the gas must be about $\langle n\rangle \sim 10^{3} \mathrm{~cm}^{-3}$. At a galactocentric radius of about one hundred parsecs this gas will be tidally disrupted unless it is adjacent and bound to a denser molecular cloud. This gas forms a non-cloudy medium, just as the atomic gas in the solar neighborhood is partly in gravitationally-bound clouds and partly in a more diffuse, non-cloudy medium. The entire "nuclear disk" feature (Liszt and Burton, 1980) appears to be made of such material.

The absence of gas at permitted velocities in Figure 1 can only be explained by the concentration of the gas into an elongated structure which is not azimuthally symmetric. The term "nuclear disk" is a misnomer, because this material actually appears to be in a dust lane. Such dust lanes are common features in galaxies having a stellar bar which gives rise to a nonaxisymmetric gravitational potential. Thus the confinement of gas to a restricted velocity range can be used to argue that our Galaxy contains a stellar bar that is sufficiently triaxial to produce shocks in the molecular gas which sweep most of it into a dust lane. Dense clouds in such a lane must move in elongated orbits about the galactic center, a feature of this model which readily explains the "forbidden" velocities observed in the gas.

The dust lane contains both gas in clouds and gas not in clouds. The motion of bound clouds through the diffuse medium will produce shocks which may produce 
turbulence inside the clouds and energize some of the filamentary centimeter-wave radio structures observed near the galactic center.

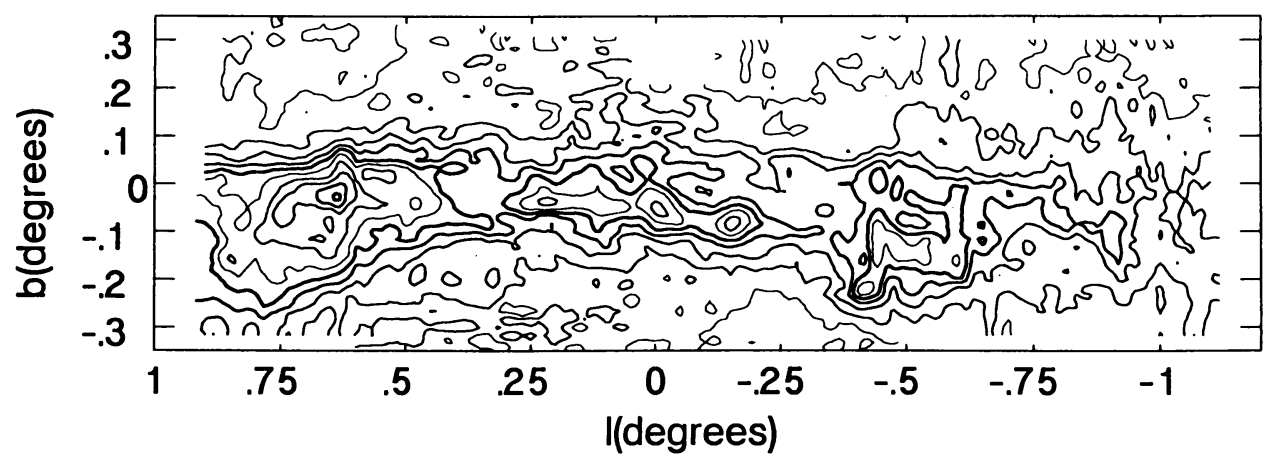

Fig. 3. Integrated ${ }^{13} \mathrm{CO}$ flux in the inner $2^{\circ}$ of the Galaxy. Contours are equally spaced at intervals of $40 \mathrm{Kkm} \mathrm{s}^{-1}$.

The radiative transfer of molecular lines in such a medium may be significantly different from that in clouds. In particular, the mean optical depth may be less because of large velocity gradients. The galactic center may therefore emit more $\mathrm{CO}$ radiation per unit mass than the rest of the galaxy. Note that the arguments presented above are sufficiently general that they apply to any galaxy, and that the CO emission from the centers of all galaxies (especially active galaxies) may arise in "non-cloudy" molecular gas.

We thank Win Bent, Dennis Mumma, and Aaron Krahnert for their contributions to this work.

\section{REFERENCES}

Bally, J., Stark, A. A., Wilson, R. W., and Henkel, C. 1987 Ap. J. Suppl., 65, 13.

Bally, J., Stark, A. A., Wilson, R. W., and Henkel, C. 1988 Ap. J., 324, 223.

Bania, T. M., Stark, A. A. and Heiligman, G. M. 1986 Ap. J., 307, 350.

Güsten, R., Genzel, R., Wright, M. C. H., Jaffe, D. T., Stutzki, J., Harris, A. I. 1987 Ap. J., 318, 124.

Heiligman, G. M. 1987 Ap. J., 314, 747.

Linke, R. A., Stark, A. A. and Frerking, M. A. 1981 Ap. J., 243, 147.

Liszt, H. S., and Burton, W. W. 1980 Ap. J., 236, 779.

Stark, A. A., and Bania, T. M. 1986 Ap. J. (Letters), 306, L17. 